\title{
«Postweaning Multisystemic Wasting Syndrome» (PMWS) und «Porcine Dermatitis and Nephropathy Syndrome» (PDNS) in der Schweiz in den Jahren $2003-2006$
}

\author{
Welti, S ; Sydler, T ; Wiederkehr, D ; Pospischil, A ; Hässig, M ; Bürgi, E ; Sidler, X
}

\begin{abstract}
In Switzerland postweaning multisystemic wasting syndrome (PMWS), caused by porcine circovirus type 2, was detected for the first time in 2001. To comprise the PMWS epizooty in $2003-2006$ retrospectively, individual animals were diagnosed according to internationally accepted criteria and temporal and regional patterns of the epizooty were reconstructed. Occurrence of PMWS was predominantly in regions with a high frequency of swine farms (central and eastern Switzerland). Apparently it was spread to other, less affected regions, through trade of infected fattening pigs. Concurrently, disease was found in different establishments of production. Affected were mainly weaners or fattening pigs. In $40 \%$ of the breeding farms and in $25 \%$ of the fattening farms mortality rate was higher than 5 $\%$. Starting in 2003, also a higher frequency of porcine dermatitis and nephropathy syndrome (PDNS) diseased pigs was diagnosed. In the years 2004 to 2006 they accounted for about $10 \%$ of the diagnosed PCV2-associated diseases. Besides the characteristic skin- and kidney lesions approximately half of the PDNS cases showed wasting and lymphoid lesions with high quantities of PCV2 antigen. We termed these mixed forms PMWS-PDNS-hybrid forms. In der Schweiz wurde das «porcine multisystemic wasting syndrome»(PMWS), verursacht durch das porzine Circovirus Typ 2 (PCV2), im Jahr 2001 zum ersten Mal beschrieben. Um die darauf folgende PMWS-Epizootie retrospektiv erfassen zu können, wurden die PMWS-Diagnosen nach Vorgaben des 6. EU-Rahmenprogramms aufgearbeitet und die zeitliche und regionale Entwicklung der Epizootie rekonstruiert. PMWS trat vor allem in Regionen mit hoher Schweinedichte (Zentral- und Ostschweiz) auf und wurde über den Verkauf von infi zierten Mastferkeln auch in Regionen mit geringerer Schweinedichte verbreitet. Erkrankungen traten zeitgleich in allen Produktions- und Haltungssystemen auf. Betroffen waren vor allem Absetzferkel aus Ferkelaufzuchtbetrieben sowie Zucht- und Mastbetriebe. Bei rund $40 \%$ der betroffenen Zucht- und bei $25 \%$ der Mastbetriebe lag die Mortalitätsrate über $5 \%$. Ab 2003 wurde auch das «porcine dermatitis and nephropathy syndrome »(PDNS) vermehrt diagnostiziert, welches in den Jahren $2004-2006$ rund $10 \%$ der diagnostizierten PCV2-assoziierten Erkrankungen ausmachte. Etwa die Hälfte der PDNS-Fälle zeigte neben den charakteristischen Haut- und/oder Nierenläsionen auch Kümmern und lymphatische Läsionen mit Präsenz von hohen PCV2-Antigenmengen. Diese bezeichneten wir als PMWS-PDNS-Mischformen.
\end{abstract}

DOI: https://doi.org/10.1024/0036-7281/a000378

Other titles: Postweaning multisystemic wasting syndrome (PMWS) and porcine dermatitis and nephropathy syndrome (PDNS) in Switzerland in the years $2003-2006$

Posted at the Zurich Open Repository and Archive, University of Zurich

ZORA URL: https://doi.org/10.5167/uzh-73986

Journal Article

Accepted Version 
Originally published at:

Welti, S; Sydler, T; Wiederkehr, D; Pospischil, A; Hässig, M; Bürgi, E; Sidler, X (2012). «Postweaning Multisystemic Wasting Syndrome» (PMWS) und «Porcine Dermatitis and Nephropathy Syndrome» (PDNS) in der Schweiz in den Jahren 2003 - 2006. Schweizer Archiv für Tierheilkunde, 154(10):417-427. DOI: https://doi.org/10.1024/0036-7281/a000378 
1 Postweaning Multisystemic Wasting Syndrome (PMWS) und Porzines Dermatitis

2 Nephropathie Syndrom (PDNS) in der Schweiz in den Jahren 2003-2006

3

4 Schlüsselwörter: PMWS, PDNS, PCV2, Epizootie, Schwein Bestandesmedizin der Universität Zürich

\section{Zusammenfassung} PDNS-Mischformen.
S. Welti ${ }^{1}$, T.Sydler ${ }^{2}$,
D. D. Wiederkehr²,
A. Pospischil2, M. Hässig ${ }^{3}$,
E. Buergi ${ }^{1}, \mathrm{X}$. Sidler ${ }^{1}$

1Departement für Nutztiere, Abteilung Schweinemedizin, ${ }^{2}$ Institut für Veterinärpathologie und ${ }^{3}$ Departement für Nutztiere, Abteilung Ambulanz und

In der Schweiz wurde das "porcine multisystemic wasting syndrome" (PMWS), verursacht durch das porzine Circovirus Typ 2 (PCV2), im Jahr 2001 zum ersten Mal beschrieben. Um die darauf folgende PMWS-Epizootie retrospektiv erfassen zu können, wurden die PMWS-Diagnosen nach Vorgaben des $6 . \mathrm{EU}$ Rahmenprogramms aufgearbeitet und die zeitliche und regionale Entwicklung der Epizootie rekonstruiert. PMWS trat vor allem in Regionen mit hoher Schweinedichte (Zentral- und Ostschweiz) auf und wurde über den Verkauf von infizierten Mastferkeln auch in Regionen mit geringerer Schweinedichte verbreitet. Erkrankungen traten zeitgleich in allen Produktions- und Haltungssystemen auf. Betroffen waren vor allem Absetzferkel aus Ferkelaufzuchtbetrieben sowie Zuchtund Mastbetriebe. Bei rund 40\% der betroffenen Zucht- und bei $25 \%$ der Mastbetriebe lag die Mortalitätsrate über 5\%. Ab 2003 wurde auch das porzine Dermatitis Nephropathie Syndrom (PDNS) vermehrt diagnostiziert, welches in den Jahren 2004-2006 rund 10\% der diagnostizierten PCV2-assoziierten Erkrankungen ausmachte. Etwa die Hälfte der PDNS-Fälle zeigte neben den charakteristischen Haut- und/oder Nierenläsionen auch Kümmern und lymphatische Läsionen mit Präsenz von hohen PCV2-Antigenmengen. Diese bezeichneten wir als PMWS- 
34 Postweaning multisystemic wasting syndrome (PMWS) and porcine dermatitis and 35 nephropathy syndrome (PDNS) in Switzerland in the years 2003-2006

Summary

In Switzerland postweaning multisystemic wasting syndrome (PMWS), caused by porcine circovirus type 2, was detected for the first time in 2001 . To comprise the PMWS epizooty in 2003-2006 retrospectively, individual animals were diagnosed according to internationally accepted criteria and temporal and regional patterns of the epizooty were reconstructed. Occurrence of PMWS was predominantly in regions with a high frequency of swine farms (central and eastern Switzerland). Apparently it was spread to other, less affected regions, through trade of infected fattening pigs. Concurrently, disease was found in different establishments of production. Affected were mainly weaners or fattening pigs. In $40 \%$ of the breeding farms and in $25 \%$ of the fattening farms mortality rate was higher than $5 \%$. Starting in 2003 , also a higher frequency of porcine dermatitis and nephropathy syndrome (PDNS) diseased pigs was diagnosed. In the years 2004 to 2006 they accounted for about $10 \%$ of the diagnosed PCV2-associated diseases. Besides the characteristic skin- and kidney lesions approximately half of the PDNS cases showed wasting and lymphoid lesions with high quantities of PCV2 antigen. We termed these mixed forms PMWS-PDNShybrid forms.

Keywords: PMWS, PDNS, PCV2, epizooty, pig 
Das „postweaning multisystemic wasting syndrome“ (PMWS) wurde 1991 zum ersten Mal in Westkanada beobachtet und 1996 als schweinespezifisches Krankheitsbild beschrieben (Clark, 1997; Harding, 1997; Harding und Clark, 1997b). Heute kommt PMWS in allen schweineproduzierenden Ländern der Welt vor. Tierverluste, reduzierte Tageszunahmen und Sekundärerkrankungen verursachen grosse finanzielle Verluste, die nach Schätzungen für die Europäische Union (EU) zwischen 562 und 900 Millionen Euro pro Jahr betragen (Armstrong, 2004; http//v2.mlc.org.uk.; Segales et al., 2006b).

Anfänglich traten die ersten klinischen Symptome vor allem bei Absetzferkeln und jungen Mastschweinen im Alter zwischen 4 und 14 Wochen auf (Harding, 1997; Harding et al., 1998; Allan und Ellis, 2000a; Rodríguez-Arrioja et al., 2002). Heute tritt PMWS auch bis zur 20. Lebenswoche oder noch später auf (Charreyre et al., 2008). Das unspezifische Leitsymptom „Kümmern“ beinhaltet progressive Abmagerung ohne begleitende Anorexie und ein struppiges Haarkleid. Häufige zusätzliche Symptome sind Dyspnoe, Diarrhoe, Anämie, Ikterus und/oder eine Vergrösserung der peripheren Lymphknoten (Harding und Clark, 1997b; Segales et al., 1997; Harding et al., 1998; Madec et al., 2000; 2008). Für akute Ausbrüche werden Morbiditätsraten von 4-60 \% und Letalitätsraten von 4-40\% beschrieben (Harding und Clark, 1997b; Muirhead, 2002; Rodríguez-Arrioja et al., 2002). PMWS ist immer mit einer Infektion mit dem porzinen Circovirus Typ 2 (PCV2) vergesellschaftet. Das Virus zirkulierte aber schon in der Schweinepopulation bevor PMWS vermehrt auftrat (Walker et al., 2000; Rodriguez-Arrioja et al., 2003b; Grierson et al., 2004a; Staebler et al., 2005). Bis jetzt ist unklar, ob zusätzliche infektiöse oder nichtinfektiöse Faktoren oder eine Steigerung der Virulenz infolge Mutation von PCV2 zu den epizootischen Krankheitsausbrüchen geführt haben (Opriessnig et al., 2007; Wiederkehr et al., 2009). In der Schweiz wurde PMWS erstmals im Jahre 2001 beschrieben (Borel et al., 2001). Zu dieser Zeit waren PCV2-Infektionen keine bedeutende Ursache für Kümmern nach dem Absetzen, obwohl Antikörper gegen PCV2 bei Absetzferkeln häufig gefunden werden konnten (Staebler et al., 2004). Seit Anfang 2003 wurden in der Routinediagnostik des Institutes für Veterinärpathologie Zürich vereinzelt PMWSDiagnosen gestellt und gegen Ende 2003 wurden die ersten PMWS- 
102 Bestandesprobleme verzeichnet (Sydler und Buergi, 2004). Damit begann die 103 PMWS-Epizootie in der Schweiz (Wiederkehr et al., 2009). Seit 2005 wurde die 104 PMWS-Diagnostik am Institut für Veterinärpathologie standardisiert und nach den 105 Vorgaben des 6. EU Rahmenprogramms (www.pcvd.org) und nach Sorden (2000) 106 durchgeführt. Beurteilt wurden: 1.) Exterieur der Tiere und charakteristische klinische 107 Symptome, 2.) charakteristische histologische Läsionen im lymphatischen Gewebe 108 (periphere und mesenteriale Lymphknoten, Tonsillen, Milz und Peyersche Platten) 109 und 3.) der immunhistochemische Nachweis (IHC) einer mittelgradigen (++) bis 110 hochgradigen (+++) PCV2-Antigenmenge in den Läsionen der lymphatischen 111 Organe. Von einer PMWS-Einzeltierdiagnose lässt sich nicht direkt auf die PMWS112 Bestandessituation schliessen. Die vom 6. EU Rahmenprogramm und von der 113 American Association of Swine Veterinarians vorgeschlagenen Eckpfeiler einer 114 PMWS-Bestandesdiagnose stützen sich auf die PMWS-Diagnose am einzelnen Tier 115 und den Anstieg der Herdenmortalität über einen festgelegten Wert 116 (http://www.aasp.org/aasv/position-PCVAD.htm, 4. Februar 2007); Opriessnig et al., 117 2007; www.pcvd.org). Andere Ursachen für die erhöhte Mortalität müssen 118 ausgeschlossen werden.

119 Im Jahr 2004 wurde darauf hingewiesen, dass in der Schweiz auch das "porcine 120 dermatitis nephropathy syndrome" (PDNS) vorkommt (Sydler und Buergi, 2004). 121 Dieses Krankheitsbild wurde 1993 in England zum ersten Mal beschrieben (Smith et 122 al., 1993) und tritt heute weltweit, vor allem bei Mastschweinen zwischen 20 und 95 123 kg Körpergewicht auf (Smith et al., 1993; White und Higgins, 1993; Helie et al., 1995; 124 Allan et al., 2000b; Ritzmann et al., 2005). Meistens sind nur einzelne Tiere eines 125 Bestandes betroffen, die aber häufig einen schnellen und fatalen Krankheitsverlauf 126 zeigen (Drolet, 1999; Thomson et al., 2002). Hämorrhagische Hautveränderungen 127 sind das auffälligste klinische Symptom, die aber spontan wieder abheilen können 128 (Smith et al., 1993; Ritzmann et al., 2005). Auf Grund einer diffusen exsudativen 129 Glomerulonephritis können die Tiere sterben (Smith et al., 1993; White und Higgins, 130 1993; Segales et al., 1998; Thomson et al., 2002). Sowohl Hautveränderungen als 131 auch Nierenläsionen erinnern an die Symptome der Schweinepest.

132 Ziel dieser Arbeit ist es, die PMWS-Epizootie in der Schweiz zwischen 2003 bis 2006 133 zeitlich und regional zu rekonstruieren und unter Anwendung von international 134 anerkannten Kriterien für die PMWS-Einzeltierdiagnose zu dokumentieren. Mittels 135 schriftlicher Befragung der Landwirte von betroffenen Beständen wurde versucht 
136 einen qualitativen Eindruck von PMWS auf Bestandesebene zu erhalten und die nach dem Erstausbruch getroffenen Massnahmen zu erfassen. Handelsbeziehungen von betroffenen Betrieben wurden überprüft, um die Rolle des Tierhandels bei der Ausbreitung von PCV2-Infektionen abschätzen zu können. Bei allen Untersuchungen wurde auch PDNS mitberücksichtigt.

Material und Methoden

Aufarbeitung von Sektionsdaten

Der immunhistochemische (IHC) Nachweis einer PCV2-Infektion in den lymphatischen Organen wurde in den Jahren 2003-2006 in der Schweiz ausschliesslich am Institut für Veterinärpathologie durchgeführt. Es wurden Organproben aus dem eigenen Sektionsgut, aber auch eingeschickte Organproben von diagnostischen Laboratorien oder Bestandestierärzten (Hofsektionen) untersucht. Mit Hilfe der elektronischen Datenbank des Institutes wurden alle Fälle mit einem positiven IHC-Befund für PCV2 ermittelt, um anhand von schriftlichen Angaben zu Alter und Gewicht aus Untersuchungsprotokollen und einer erneuten histologischen und immunhistologischen Gewebebegutachtung einheitlich beurteilt werden zu können. Hierfür gewährten uns auch externe diagnostische Laboratorien (Diagnostik Labor Bassersdorf GmbH, Bassersdorf; Diavet Labor AG, Bäch; Institut

156 für Klinische Mikrobiologie und Immunologie (IKMI), St. Gallen; Institut für

157 Tierpathologie der Universität Bern) Einsicht in Dokumente und stellten uns

158 Gewebeschnitte und Gewebeblöcke zur Verfügung. Zusätzlich wurde in der Datenbank nach Fällen mit exsudativer Glomerulonephritis gesucht.

162 Mehrheitlich standen für die histologische und IHC Aufarbeitung eines Falles 163 mehrere lymphatische Gewebe (periphere und mesenteriale Lymphknoten, Milz, 164 Tonsillen und lleum mit Peyerschen Platten) und oft auch Nieren zur Verfügung. Die Herstellung der Paraffinblöcke, die Schnittherstellung und die Hämatoxilin-Eosin (HE)

166 Färbung erfolgten nach Standardverfahren (Staebler et al., 2005; Wiederkehr et al., 167 2009). Für den IHC Nachweis von PCV2-Antigen wurde der monoklonale Antikörper 168 F217 verwendet (McNeilly et al., 2001). Die IHC Reaktion wurde im Färbeautomaten 169 (Dako Autostainer) durchgeführt (Wiederkehr et al.2009). 
170 Die HE-gefärbten Schnitte der lymphatischen Gewebe wurden auf Vorhandensein 171 von lymphatischer Depletion, Infiltration mit histiozytären und epitheloiden Zellen 172 sowie mehrkernigen Riesenzellen und auf das Vorhandensein von 173 intrazytoplasmatischen basophilen Einschlusskörperchen und Nekrosen untersucht 174 (Sorden, 2000; Rodríguez-Arrioja et al., 2002; www.pcvd.org). Bei den Nieren wurde 175 speziell auf das Vorliegen einer Glomerulonephritis geachtet.

176 Die IHC Beurteilung erfolgte in fünf Stufen: negativ (-; keine IHC-positiven Zellen 177 erkennbar), fraglich positiv (+/-; nur vereinzelt positive Zellen pro Schnitt), 178 geringgradig positiv (+; vereinzelte meist zentrofollikulär gelegene kleine, positive 179 Zellgruppen oder vermehrt positive Einzelzellen), mittelgradig positiv (++; 180 regelmässig auftretende zentrofollikulär und/oder im Randsinusgebiet liegende, 181 grössere positive Zellgruppen) und hochgradig positiv (+++; zusätzlich zu ++ sehr 182 viele Einzelzellen oder Zellgruppen bis nahezu alle Zellen positiv). Das am stärksten 183 betroffene lymphatische Gewebe definierte die IHC Beurteilung des Einzeltieres. Die 184 Beurteilung eines mittelgradigen (++) Antigengehaltes erfolgte nach den Vorgaben des 6. EU Rahmenprogramms (www.pcvd.org).

PMWS- und PDNS-Diagnose

188 Die Diagnose „PMWS“ wurden nach den Vorgaben des 6. EU-Rahmenprogramms 189 (Sorden, 2000; www.pcvd.org) mit Hauptgewicht auf der histologischen und IHC 190 Beurteilung gestellt. Ein Tier mit geringgradiger lymphozytärer Depletion und 191 fraglichem (+/-) oder geringgradigem (+) PCV2-Antigengehalt wurde als subklinisch 192 infiziert eingestuft (Opriessnig et al., 2007; Wiederkehr et al., 2009). PDNS-

193 Diagnosen wurden histologisch anhand einer akuten oder chronisch rezidivierenden 194 diffusen exsudativen Glomerulonephritis gestellt (Smith et al., 1993; Helie et al., 195 1995; Segales et al., 2005).

197 Herkunftsbetriebe mit PMWS-Einzeltieren

198 Die allgemeinen Angaben zu den Herkunftsbetrieben stammen aus der Datenbank 199 der SUISAG (Geschäftsbereich Gesundheit, SGD ${ }^{\circledR}$ ). Um die Rolle von 200 Handelsbeziehungen zwischen Herkunftsbetrieben zu Beginn und während der 201 PMWS-Epizootie beurteilen zu können, wurden Vermarktungsorganisationen 202 bezüglich des Tierhandels der betroffenen Betriebe befragt. Anhand der 
203 Untersuchungszeitpunkte und der Betriebsadressen wurden die Herkunftsbetriebe

204 zeitlich und geographisch erfasst und in Karten eingetragen.

206 Befragungen in Herkunftsbetrieben mit PMWS-Einzeltieren aus den Jahren 207 2005/2006

208 Im Januar 2008 wurden Fragebögen an 259 Betriebsleiter von Herkunftsbetrieben 209 mit PMWS-Tieren verschickt, für die in den Jahren 2005/06 eine PCV2-Infektion 210 diagnostiziert wurde. Alle Fragen mussten retrospektiv für die Jahre 2005 und 2006 211 beantwortet werden. In einem ersten Teil des Fragebogens wurden Fragen zu 212 Betriebsstruktur, Betriebsgrösse und Betriebsführung gestellt. Der zweite Teil war nur 213 an Betriebe gerichtet, die in den Jahren 2005/06 nach eigener Einschätzung ein 214 PMWS-Bestandesproblem hatten. Diese Fragen waren primär auf die 215 Alterskategorien Absetzferkel und Mastschweine, die Erfassung des klinischen 216 Bildes, der Morbidität und Mortalität zum Zeitpunkt des Erstauftretens von PMWS 217 und/oder PDNS im Betrieb und nachfolgende Lösungsansätze ausgerichtet. Als 218 Betriebe mit einem PMWS-Bestandesproblem wurden Betriebe definiert, welche 219 charakteristische klinische Symptome und erhöhte Tierverluste beklagten und 220 mindestens eine positiven PMWS-Diagnose aufwiesen.

222 Datenverarbeitung

223 Das Datenmanagement wurde mit Filemaker Pro 8 und die statistischen 224 Auswertungen mit StatView ${ }^{\circledR} 5.0$ für Windows (SAS Institute Inc. Copyright@) 1992225 1998) durchgeführt. Ein P-Wert von < 0.05 wurde als signifikant betrachtet. Die 226 Koordinaten der Standorte der Herkunftsbetriebe wurden mit TwixTel und TwixRoute 227 (Twix AG, Version 38, Mai 2008) adressgenau ermittelt. Die Karten wurden mit 228 ArcGis 9, ArcView $® 9.2$ (Environmental Systems Research Institute (ESRI), 229 Redlands, USA) erstellt. Räumliche und räumlich-zeitliche statistische Auswertungen 230 wurden mit SATScan ${ }^{\mathrm{TM}}$ 7.0.3 durchgeführt. Es wurde je eine räumliche und eine 231 räumlich-zeitliche Analyse auf erhöhtes Risiko mit dem Poisson-Modell mit 999 232 Iterationen gerechnet. Die maximale Fenstergrösse betrug jeweils $50 \%$.

\section{Ergebnisse}


237 In den Jahren 2003 bis 2006 wurden anhand von Gewebeproben 538 Fälle als 238 PCV2-infiziert diagnostiziert. Davon wurden 189 (35.1\%) Tierkörper und 8 (1.5\%) 239 direkt an das Institut für Veterinärpathologie eingesandte Organeinsendungen sowie $240216(40.1 \%)$ Tierkörper und 125 (23.2\%) Organeinsendungen von andern 241 Diagnostiklaboratorien untersucht. Bei der Neubeurteilung konnten in 14 (2.6\%) 242 Fällen keine IHC-positiven Strukturen mehr nachgewiesen werden. Dies spricht für 243 eine fragliche (+/-) bis geringgradige (+) Antigenmenge bei der Erstbeurteilung. Mehr 244 als die Hälfte dieser Tiere zeigten aber histologische Läsionen in den lymphatischen 245 Organen, die mit PMWS vereinbar waren. Die lymphatische Depletion war bei einem 246 Tier hochgradig, bei den anderen leichtgradig ausgeprägt.

247 Von den 538 Schweinen wurde bei 393 (73.0\%) mit mittelgradigem (++) oder 248 hochgradigem (+++) PCV2-Antigengehalt eine PMWS-Diagnose gestellt (Tab. 1). Die 249 lymphatischen Läsionen waren meist deutlich bis hochgradig ausgeprägt. Bei 49 $250(12.5 \%)$ dieser 393 PMWS-Einzeltierfälle waren intrazytoplasmatische 251 Einschlusskörperchen vorhanden, wobei der Grossteil zusammen mit hohem PCV2252 Antigengehalt auftrat. Nekrosen in den lymphatischen Organen traten bei 44 (11.2\%) 253 PMWS-Einzeltierfällen auf. Bei 22 (5.6\%) wurde zusätzlich zu PMWS auch PDNS 254 diagnostiziert. Bei 93 (17.3\%) der 538 untersuchten Tiere konnte nur eine geringe (+) 255 Antigenmenge gefunden werden. Davon waren 16 (17.2\%) PDNS-Tiere und 77 256 (82.8\%) subklinisch mit PCV2 infizierte Tiere (Tab. 1). Eine fragliche (+/-) 257 Antigenmenge wiesen 38 (7.1\%) von 538 Tieren auf, wovon 5 (13.2 \%) PDNS-Fälle 258 und 33 (86.8\%) subklinisch infiziert waren. Auch von diesen Fällen zeigte ein grosser 259 Anteil lymphatische Läsionen. Die lymphozytäre Depletion reichte von leicht- bis 260 hochgradig (Tab. 2).

261 Nach Angaben aus den Anamnesen waren die PMWS-Tiere 10.5 \pm 3.8 Wochen alt $262(n=167)$ und 14.6 $\pm 8.8 \mathrm{~kg}$ schwer $(n=276)$. Die PDNS-Tiere waren 17.0 \pm 4.2 Wochen 263 alt $(n=9)$ und wogen $44.1 \pm 20.7 \mathrm{~kg}(n=19)$. Tiere mit einer Mischform (PMWS und 264 PDNS) hatten ein Alter von 17.5 \pm 4.2 Wochen $(n=6)$ und ein Gewicht von 29.8 \pm 11.9 $265 \mathrm{~kg}(\mathrm{n}=13)$. Die PMWS-Tiere waren signifikant leichter und jünger als die an PDNS 266 und an einer Mischform erkrankten Tiere $(p<0.001)$. Die an einer Mischform 267 erkrankten Tiere waren zwar gleich alt, aber signifikant leichter als die PDNS-Tiere $268 \quad(p<0.001)$.

269 Die ersten 2 PDNS-Fälle mit Nieren- und Hautläsionen im Archiv stammten aus dem 270 Jahr 1989 (nicht publiziert). Je ein Fall ohne Hautläsionen stammte aus den Jahren 
1991, 1993 und 1996 und 2 Fälle aus dem Jahr 1995. Weitere PDNS-Fälle wurden erst ab 2003 verzeichnet. Somit haben mit dem Beginn der PMWS-Epizootie nicht nur die PMWS-Einzeltierfälle, sondern auch die zur Abklärung eingeschickten PDNS-

274 Fälle und Mischformen zugenommen. Diese machten in den Jahren 2004-2006 rund 10\% der diagnostizierten PCV2-assoziierten Erkrankungen aus (Abb. 1).

\section{Epidemiologische Daten}

278 Herkunftsbetriebe mit PMWS-Einzeltieren

279 Die Schweine mit einer PMWS-Diagnose stammten aus 258 verschiedenen 280 Betrieben. Aus 24 Betrieben wurden mehrere Tiere zur Untersuchung eingeschickt. 281 Die Anzahl der Herkunftsbetriebe hatte pro Jahr von 2003 bis 2006 laufend 282 zugenommen (Abb. 2). Die meisten PMWS-Tiere stammten aus 118 Betrieben aus 283 dem Kanton Luzern mit der grössten Schweinedichte ( $\varnothing 30.9$ Muttersauen pro km²) 284 und aus 30 Betrieben im Kanton Aargau ( $\varnothing 6.3$ Muttersauen pro $\mathrm{km}^{2}$ ). In der 285 Ostschweiz mit der zweitgrössten Schweinedichte in der Schweiz (Kantone Thurgau, 286 St. Gallen, Appenzell Innerrhoden, Appenzell Ausserrhoden, Schaffhausen) 287 befanden sich 65 Betriebe.

288 Die meisten Fälle aus dem Kanton Luzern stammten aus einer räumlichen 289 Anhäufung (Räumlicher Cluster 2003-2006, Radius=14.3 km, Relatives Risiko= 2.9; $290 \mathrm{p}<0.001$ ) im Norden des Kantons (Abb.3). Eine räumlich-zeitliche Häufung von Juni 2912005 bis November 2006 befand sich ebenfalls im nördlichen Teil des Kantons, ist 292 aber im Vergleich zum räumlichen Cluster um etwa $1 \mathrm{~km}$ nach Osten verschoben 293 (Räumlich-zeitlicher Cluster 2005-2006, Radius=16.2 km, Relatives Risiko=6.3; $294 \mathrm{p}<0.001, \mathrm{Abb} .3)$. Auffallend zahlreiche betroffene Mastbetriebe in der Ost- und 295 Westschweiz wurden von Zuchtbetrieben aus dem Kanton Luzern beliefert.

296 Retrospektive Untersuchungen der Handelsbeziehungen von Betrieben mit PMWS297 Tieren wurden aufgrund einer Befragung von Vermarktungsorganisationen und der 298 Auswertung von SGD-Daten bezüglich Gesundheitsstatus in den Jahren 2003-2006 299 analysiert. Die Analyse ergab, dass sich unter den Beständen, die 2003/04 Tiere zur 300 PMWS Abklärung eingesandt hatten, keine Kern- oder Vermehrungszuchtbetriebe 301 befanden. In Kern- und Vermehrungszuchtbetrieben wurden erstmals 2005/06 302 PMWS-Tiere diagnostiziert. PMWS konnte fast zeitgleich bei Mastferkelproduzenten 303 und in Mastbeständen von mehreren Vermarktungsorganisationen nachgewiesen 304 werden. 
305 Die Betriebsstrukturen der Herkunftsbetriebe waren vielfältig: So kamen 306 Zuchtbetriebe $(n=106)$, Zucht-Mastbetriebe $(n=15)$, Abferkelbetriebe $(n=22)$ von

307 Ringbetrieben mit arbeitsteiliger Ferkelproduktion, Ferkelaufzuchtbetriebe $(n=10)$, 308 Ferkelaufzucht-Mastbetriebe $(n=5)$ und Mastbetriebe $(n=103)$ im Untersuchungsgut 309 vor. Unter Berücksichtigung der Häufigkeit der verschiedenen Betriebsarten in der 310 Schweiz waren Ferkelaufzuchtbetriebe sehr viel häufiger betroffen als Zucht- und 311 Mastbetriebe. Zuchtbetriebe mit mehr als 50 Muttersauen, oder Mastbetriebe mit 312 mehr als 500 Mastschweinen waren signifikant häufiger betroffen, als Zuchtbetriebe 313 mit weniger als 50 Muttersauen oder Mastbetriebe mit weniger als 500 314 Mastschweinen $(p<0.001)$.

316 Betriebe mit einem PMWS-Bestandesproblem in den Jahren 2005/06

317 Von 259 verschickten Fragebögen wurden 184 zurückgesandt (Rücklaufquote 71\%). 318 Davon stammten 139 aus Betrieben, welche die Kriterien für ein PMWS319 Bestandesproblem erfüllten. Aus 9 der 139 PMWS-Problembetriebe stammten auch 320 Tiere mit einer PDNS-Diagnose. Bei 30 Betrieben mit einem Bestandesproblem 321 fehlte ein gesicherter Nachweis von PMWS. Allerdings wurden aus Betrieben 322 meistens nur ein bis zwei Tiere ( $\varnothing 1.2$ Tiere pro Betrieb) zur Untersuchung 323 eingeschickt.

324 Auf 66 (47.5\%) Betrieben wurden Absetzferkel, auf 45 (32.4\%) ausschliesslich 325 Mastschweine und auf 28 (20.1\%) Absetzferkel und Mastschweine gehalten. Bei den 326 zuletzt genannten Betrieben waren in 17 Betrieben hauptsächlich die Absetzferkel, in 3272 Fällen hauptsächlich die Mastschweine und in 9 Fällen beide Altersklassen 328 betroffen. Die PMWS-Leitsymptome Kümmern und Abmagerung wurden sowohl bei 329 den Absetzferkeln als auch bei den Mastschweinen als häufigste Symptome und 330 Durchfall als zweithäufigstes Symptom beobachtet. PDNS-ähnliche 331 Hautveränderungen wurden zwar häufiger bei Mastschweinen (39.7\%), aber 332 erstaunlich häufig auch bei Absetzferkeln (12.8\%) von den Betriebsleitern erwähnt 333 (Tab. 3). Zum Zeitpunkt des Erstausbruchs von PMWS erkrankten in 61.7\% der 334 Mastferkelproduktionsbetriebe mehr als 5\% der Absetzferkel und in 39.4\% starben 335 mehr als 5\%. In 49.3\% der Mastbetriebe erkrankten mehr als 5\% der Mastschweine 336 und in $27.4 \%$ starben mehr als $5 \%$ (Tab. 4). Auf vielen Betrieben verbesserte sich die 337 PMWS-Situation vom Erstausbruch bis zum Frühling 2008, wobei alle Landwirte in 338 Anlehnung an den 20-Punkte-Plan von Madec et al., (1999) Massnahmen ergriffen 
339 hatten, um den Infektionsdruck auf dem Betrieb zu senken. In 90.4\% der Betriebe mit 340 Absetzferkeln erkrankten weniger Tiere und in $66.6 \%$ der Betriebe war die 341 Sterblichkeit auch geringer. Ein ähnliches Bild zeigte sich auf den Mastbetrieben. In $34256.2 \%$ der Betriebe erkrankten weniger und in 39.7\% der Betriebe starben weniger 343 Tiere. Nur zwei Betriebe mit Mastschweinen gaben erhöhte Morbiditäts- und 344 Letalitätsraten an. Begleiterkrankungen wurden selten erwähnt. Dabei wurden bei

345 den Absetzferkeln und bei den Mastschweinen Durchfallerkrankungen mit $L$. 346 intracellularis und E. coli als Koinfektionen am häufigsten genannt. In 97 (57.4\%) 347 Betrieben wurden zur Bekämpfung von Begleiterkrankungen die betroffenen 348 Tiergruppen peroral mit Antibiotika behandelt. Kombination von Chlortetrazyklin, 349 Tylosin und Sulfadimidin wurden in 54 (32.0\%) Betrieben und Tetrazyklin als 350 Monosubstanz in 31 (18.3\%) Betrieben am häufigsten eingesetzt.

352 Diskussion

354 PMWS wurde im Jahre 2001 erstmals in der Schweiz beschrieben (Borel et al., 2001). Damals stellte PCV2 keine bedeutende Ursache für vermehrtes Kümmern nach dem Absetzen in Schweizer Schweinezuchtbetrieben (Staebler et al., 2004) dar. Erst gegen Ende 2003 und vor allem in den Jahren 2004-2006 wurden vermehrt Schweine zur PMWS-Abklärung der Routinediagnostik zugeführt (Wiederkehr et al., 2009). Die PMWS-Epizootie entwickelte sich somit in der Schweiz später als in vielen anderen europäischen Ländern (Gresham et al., 2000; Madec et al., 2000; Vigre et al., 2005; Segales, 2007) aber ungefähr gleichzeitig wie in Schweden (Wallgren et al., 2004; 2007). In der Schweiz kommen im Gegensatz zu vielen anderen 363 schweineproduzierenden Ländern verschiedene in der Literatur beschriebene 364 Risikofaktoren für PMWS nicht vor. Zudem sind schweizerische Schweinebetriebe durchschnittlich deutlich kleiner als im übrigen Europa (Eurostat, 2007). Die Schweiz 366 ist frei von allen auf der Liste des Office International des Epizooties (OIE) 367 aufgeführten Krankheiten. Die Freiheit von PRRS wurde in den Jahren 2001-2006 mittels Stichprobenuntersuchungen bewiesen (Corbellini et al., 2006; Schwermer und 369 Sievi, 2010) und die beiden weltweit stark verbreiteten Atemwegserkrankungen die 370 Enzootische Pneumonie (EP) und die Actinobazillose wurden in der Schweiz 1995 in 371 die Liste der „zu bekämpfenden Tierseuchen“ aufgenommen. Deshalb sind 372 Impfungen gegen M. hyopneumoniae, Actinobacillus pleuropneumoniae (APP) und 
gegen PRRS gesetzlich verboten. Seit Abschluss der Flächensanierung im Jahre 2004 ist die EP-Reinfektionsrate geringer als 1\% (Staerk et al., 2007). Die porzine Parvovirose kommt in der Schweiz vor, aber in den meisten Zuchtbetrieben wird routinemässig dagegen geimpft.

377 Betroffene Tiere und Bestände stammten mehrheitlich aus Gebieten mit hoher 378 Schweinedichte (Kanton Luzern, Ostschweiz). Durch den Verkauf infizierter Masttiere 379 wurde die PCV2-Infektion auch in weniger schweinedichte Gebiete in der Schweiz 380 verbreitet. Die räumliche und zeitliche Anhäufung von PCV2 infizierten Tieren im 381 nördlichen Teil des Kantons Luzern lässt vermuten, dass das Virus auch aerogen verbreitet wird. Verglichen mit der Schweinedichte wurden auffallend wenige Tiere aus dem Kanton Bern eingesandt. Ein möglicher Grund hierfür könnte die geographische Lage der diagnostischen Laboratorien gewesen sein. IHC Untersuchungen wurden nur am Institut für Veterinärpathologie in Zürich durchgeführt und Einsendungen von verdächtigen Organproben stammten mehrheitlich aus Privatlaboratorien aus der Ost- und Zentralschweiz. Es muss davon ausgegangen werden, dass bei weitem nicht aus allen Betrieben mit einem PMWSBestandesproblem Tiere zur Untersuchung eingeschickt wurden.

390 Die ersten PMWS-Schweine aus Kern-, und Vermehrungszuchtbetrieben wurden erst 391 in den Jahren 2005/06 zur Untersuchung eingesandt. Dem kommt insbesondere 392 Bedeutung zu, weil die meisten Zuchtbetriebe (Mastferkelproduzenten) dem SGD 393 angehören und nach Reglement von AR-Betrieben Jungsauen beziehen müssen. 394 Somit spielen die Kern- und Vermehrungszuchtbetriebe zu Beginn der Epizootie für 395 die Ausbreitung von PCV2 keine Rolle. Das spätere Auftreten von PMWS in den 396 Kernzuchtbetrieben könnte dadurch erklärt werden, dass in Kernzuchtbetrieben kein 397 Tierzukauf gestattet ist und Genetikerneuerung nur durch künstliche Besamung oder 398 seltener auch durch den Zukauf von hysterotomierten Ferkeln erfolgen darf. Als 399 wahrscheinlichste Infektionsquellen für Kernzuchtbetriebe kommen daher eine 400 aerogene Erregerübertragung, eine Infektion über infiziertes Sperma oder eine 401 sukzessive Erregerakkumulation in den Betrieben in Frage. Allerdings besteht eine 402 Dunkelziffer von Betrieben, die möglicherweise PMWS-Probleme hatten, aber keine 403 Tiere eingesandt haben.

404 Die PMWS-Diagnostik am Einzeltier erfolgt hauptsächlich am toten Tier. Die 405 Definition „klinische Symptome, Läsionen in den lymphatischen Organen, 406 mindestens mittelgradiger Antigengehalt in den Läsionen“ lässt für die 
Diagnosestellung einen beachtlichen subjektiven Spielraum zu. Wichtige Kriterien zur

408 Beurteilung von „Kümmern“ sind das genaue Erfassen von Alter und Gewicht des

409 Tieres sowie die Beurteilung des Nährzustandes. Die histologische Ausprägung von

410 Läsionen und die dazu assoziierte Antigenmenge können am gleichen Tier in den

411 verschiedenen lymphatischen Organen variieren. Deshalb müssen immer mehrere

412 lymphatische Organe untersucht werden. Überdies korreliert die Ausprägung der

413 histologischen Läsionen zwar häufig, aber nicht immer, mit der detektierten PCV2-

414 Antigenmenge. Dabei muss bedacht werden, dass nicht alle Tiere zum gleichen

415 Zeitpunkt der Infektion eingeschickt werden (Opriessnig et al., 2007). Trotzdem ist

416 die Immunhistologie (IHC) der objektivste Teil der heute international anerkannten

417 Definition der PMWS-Einzeltierdiagnose, weil damit die Antigenmenge in den

418 Läsionen dokumentiert werden kann. Die IHC kann aber nicht zwischen

419 verschiedenen PCV2-Genotypen unterscheiden (Turner et al., 2009). Es gibt

420 Hinweise, dass der Genotyp PCV2a weniger pathogen ist als der Genotyp PCV2b. In

421 der Schweiz wurde seit Beginn der Epizootie fast ausschliesslich PCV2b

422 nachgewiesen (Wiederkehr et al., 2009). Sollte dies zutreffen, müssten in Zukunft

423 zusätzliche diagnostische Methoden etabliert werden.

424 Eine PMWS-Diagnose am einzelnen Tier ist nicht gleichzusetzen mit der Diagnose 425 eines PMWS-Bestandesproblems. In der internationalen Literatur wird mit einem 426 „PMWS-Fall“ häufig ein Bestandesproblem und nicht eine PMWS427 Einzeltiererkrankung bezeichnet. $\mathrm{Zu}$ einer PMWS-Bestandesdiagnose gehören 428 neben PMWS-Einzeltierdiagnosen auch vermehrtes Kümmern und erhöhte, 429 quantifizierbare Tierverluste im Bestand (Opriessnig et al., 2007). Das EU430 Rahmenprogramm (www.pcvd.org) fordert, dass bei der Bestandesdiagnostik 431 mindestens 5 Tiere zur Untersuchung eingeschickt werden. Die Diagnose ist umso 432 sicherer, je mehr Tiere untersucht werden. In den Jahren 2003-2006 wurden oft nur 433 wenige Tiere (1-2 pro Bestand) zur Untersuchung eingeschickt. Vermutlich wäre der 434 Anteil an PMWS-Betrieben noch höher gewesen, wenn mehr Tiere für die Diagnostik 435 zur Verfügung gestanden hätten.

436 Angaben zu Mortalitätsraten vom Absetzen bis zum Verkauf in die Mast werden in 437 der Schweiz leider nicht routinemässig erhoben und können daher in dieser Studie 438 nur geschätzt werden. Nach Angaben von Betriebsleitern von Betrieben mit einem 439 PMWS-Bestandesproblem scheint PMWS auch in der Schweiz verhältnismässig 440 hohe Mortalitätsraten zu verursachen, die aber nicht so hoch sind wie 
durchschnittliche Mortalitätsraten nach akuten PMWS-Ausbrüchen im Ausland (Rose

442 et al., 2003a; Lopez-Soria et al., 2005b; Nielsen et al., 2008). Da vor 2008 weder

443 eine PCV2-Mutterschutzvakzine noch eine PCV2-Ferkelvakzine zur Verfügung 444 standen, ergriffen betroffene Schweinehalter aufwändige Massnahmen wie ständiges 445 Optimieren von Managementfaktoren sowie Absondern und Ausmerzen von 446 erkrankten Tieren (Madec et al., 1999) um Produktionsverluste zu reduzieren. Diese 447 Massnahmen haben auch heute noch neben dem Einsatz von Vakzinen zentrale 448 Bedeutung. Obwohl Begleiterkrankungen nur selten erwähnt wurden und in der 449 Sektion nicht immer gezielt danach gesucht worden ist, kam zu deren Bekämpfung in 450 rund der Hälfte der befragten Betriebe eine grosse Auswahl von Antibiotika zum 451 Einsatz. Am häufigsten wurde mit tertrazyklinhaltigen Fütterungsarzneimitteln 452 behandelt. Dies widerspiegelt sich auch in den Vertriebszahlen für Antibiotika der 453 Veterinärmedizin (www.swissmedic.ch) in welchen Tetrazyklin von 2004-2007 in der 454 Nutztierhaltung um 53\% zugenommen hatte (Regula et al., 2009).

455 Parallel mit der PMWS-Epizootie stiegen auch die Anzahl PDNS-Fälle und die 456 Anzahl Mischformen sprunghaft an. Es muss davon ausgegangen werden, dass 457 PDNS in verschiedenen Krankheitsausprägungen vorkommen kann, wobei der 458 Zustand der Niere prognoserelevant ist. Ein grösserer Anteil der abgeklärten PDNS459 Fälle zeigte keine Hautläsionen. Mit Sicherheit wurden aber viele PDNS-Fälle gar 460 nicht eingeschickt, da das Krankheitsbild, wenn Hautläsionen ausgeprägt sind, recht 461 typisch und bei den Tierhaltern bekannt ist. Weil Schweine mit einer Mischform zwar 462 gleich alt wie Tiere mit reiner PDNS-Ausprägung aber signifikant leichter waren, wird 463 vermutet, dass sie zuerst an PMWS erkrankten und kümmerten, bevor sie zusätzlich 464 an PDNS erkrankten. Welche Rolle PCV2 bei der Pathogenese von PDNS spielt, ist 465 noch nicht vollständig geklärt. Bei PDNS konnten wir in den Nieren PCV2-Antigen 466 nur in geringen Mengen und nur ausserhalb der Glomerula nachweisen.

467 In der Schweiz sind routinediagnostische Methoden etabliert und sollten genutzt 468 werden, um PMWS-Bestandesprobleme, andere Ursachen für Kümmern und erhöhte 469 Mortalität oder Begleiterkrankungen fundiert abzuklären. Die Zusammenarbeit von 470 Bestandestierärzten und diagnostischen Laboratorien ist dabei essentiell, weil sich 471 PMWS/PDNS-Fälle durch eine rein klinische Untersuchung oder eine Hofsektion 472 nicht sicher von Klassischer oder Afrikanischer Schweinepest unterscheiden lassen. 473 Mit den 2008 zugelassenen PCV2-Impfstoffen scheint eine wirksame Prophylaxe zur 474 Verfügung zu stehen. Trotzdem ist auch in Zukunft eine Diagnostik von PCV2- 
475 assoziierten Erkrankungen notwendig, um unter anderem Veränderungen der 476 klinischen Symptome von PCV2-assoziierten Erkrankungen und deren Pathogenese 477 zu klären oder genetische Veränderungen des Virus rechtzeitig erfassen zu können. 478 479 480 481 482 483 484 485 486 487 488 489 490 491 492 493 494 495 496 497 498 499 500 501 502 503 504 505 506 507 508 
510

511 Tabelle 1: Immunhistochemische (IHC) Befunde und Kategorisierung der PCV2512 infizierten Tiere.

513

\begin{tabular}{|c|c|c|c|c|c|c|}
\hline IHC & $\begin{array}{l}\text { Anzahl } \\
\text { Fälle } \\
(n=538)\end{array}$ & $\begin{array}{l}\text { PMWS } \\
(n=371)\end{array}$ & $\begin{array}{l}\text { PMWS+ } \\
\text { PDNS } \\
(n=22)\end{array}$ & $\begin{array}{l}\text { PDNS } \\
(n=21)\end{array}$ & $\begin{array}{l}\text { Subklinische } \\
\text { Infektion } \\
(n=110)\end{array}$ & $\begin{array}{l}\text { Negativ } \\
(n=14)\end{array}$ \\
\hline - & 14 & & & & & 14 \\
\hline$+/-$ & 38 & & & 5 & 33 & \\
\hline+ & 93 & & & 16 & 77 & \\
\hline++ & 138 & 120 & 18 & & & \\
\hline+++ & 255 & 251 & 4 & & & \\
\hline
\end{tabular}

514

515

516

517

518

519

520

521

522

523

524

525

526

527

528

529

530

531 
532 Tabelle 2: Histologische Läsionen in Bezug zum PCV2-Antigengehalt in den 533 Läsionen der lymphatischen Organe.

534

\begin{tabular}{|c|c|c|c|c|c|}
\hline IHC & $\begin{array}{l}\text { Lymphozytäre } \\
\text { Depletion }\end{array}$ & $\begin{array}{l}\text { Histiozytäre } \\
\text { oder } \\
\text { epitheloide } \\
\text { Infiltration }\end{array}$ & $\begin{array}{l}\text { Mehrkernige } \\
\text { Riesenzellen }\end{array}$ & $\begin{array}{l}\text { Einschluss- } \\
\text { körperchen }\end{array}$ & Nekrosen \\
\hline- & 8 & 1 & 6 & & \\
\hline$+/-$ & 27 & 16 & 11 & & 4 \\
\hline+ & 68 & 38 & 15 & 1 & 7 \\
\hline++ & 138 & 101 & 61 & 4 & 37 \\
\hline+++ & 255 & 211 & 89 & 45 & 37 \\
\hline
\end{tabular}

535

536

537 
538 Tabelle 3: Klinische Symptome der Absetzferkel und Mastschweine aus PMWS539 Problembetrieben.

540

\begin{tabular}{lcc}
\hline Symptom & Anzahl Betriebe & Anzahl Betriebe \\
\hline Kümmern & $84(89.4 \%)$ & $41(56.2 \%)$ \\
Abmagerung & $72(76.6 \%)$ & $35(47.9 \%)$ \\
Diarrhoe & $73(77.7 \%)$ & $37(50.7 \%)$ \\
Anämie & $50(53.2 \%)$ & $25(34.2 \%)$ \\
Plötzliche Todesfälle & $39(41.5 \%)$ & $32(43.8 \%)$ \\
Husten & $34(36.2 \%)$ & $16(21.9 \%)$ \\
Dyspnoe & $34(36.2 \%)$ & $6(8.2 \%)$ \\
Hautveränderungen & $12(12.8 \%)$ & $29(39.7 \%)$ \\
(PDNS ähnlich) & $9(9.6 \%)$ & $3(4.1 \%)$ \\
Ödeme & $\mathbf{9 4 ( 1 0 0 \% )}$ & $\mathbf{7 3 ( 1 0 0 \% )}$ \\
\hline Total &
\end{tabular}


561 Tabelle 4: Morbidität und Mortalität von Absetzferkeln und Mastschweinen aus 562 PMWS-Problembetrieben.

563

\begin{tabular}{lcc}
\hline Morbidität \% & $\begin{array}{c}\text { Anzahl Betriebe } \\
\text { Absetzferkel (\%) }\end{array}$ & $\begin{array}{l}\text { Anzahl Betriebe } \\
\text { Mastschweine (\%) }\end{array}$ \\
\hline$<5 \%$ & $36(38.3 \%)$ & $37(50.7 \%)$ \\
$5-10 \%$ & $32(34.0 \%)$ & $16(21.9 \%)$ \\
$>10 \%$ & $25(26.6 \%)$ & $17(23.3 \%)$ \\
Keine Angabe & $1(1.1 \%)$ & $3(4.1 \%)$ \\
\hline Total & $\mathbf{9 4 ( 1 0 0 \% )}$ & $\mathbf{7 3 ( 1 0 0 \% )}$ \\
\hline
\end{tabular}

564

565

\begin{tabular}{lcc}
\hline Mortalität \% & $\begin{array}{c}\text { Anzahl Betriebe } \\
\text { Absetzferkel (\%) }\end{array}$ & $\begin{array}{c}\text { Anzahl Betriebe } \\
\text { Mastschweine (\%) }\end{array}$ \\
\hline$<5 \%$ & $57(60.6 \%)$ & $53(72.6 \%)$ \\
$5-10 \%$ & $25(26.6 \%)$ & $11(15.1 \%)$ \\
$>10 \%$ & $11(11.7 \%)$ & $7(9.6 \%)$ \\
Keine Angabe & $1(1.1 \%)$ & $2(2.7 \%)$ \\
\hline Total & $\mathbf{9 4 ( 1 0 0 \% )}$ & $\mathbf{7 3 ( 1 0 0 \% )}$ \\
\hline
\end{tabular}

566

567

568

569

570

571

572

573

574

575

576

577

578

579

580 
581

582

583

584

585

586

587

588

589

590

591

592

593

594

595

596

597

598

599

600

601

602

603

Abbildung 1:

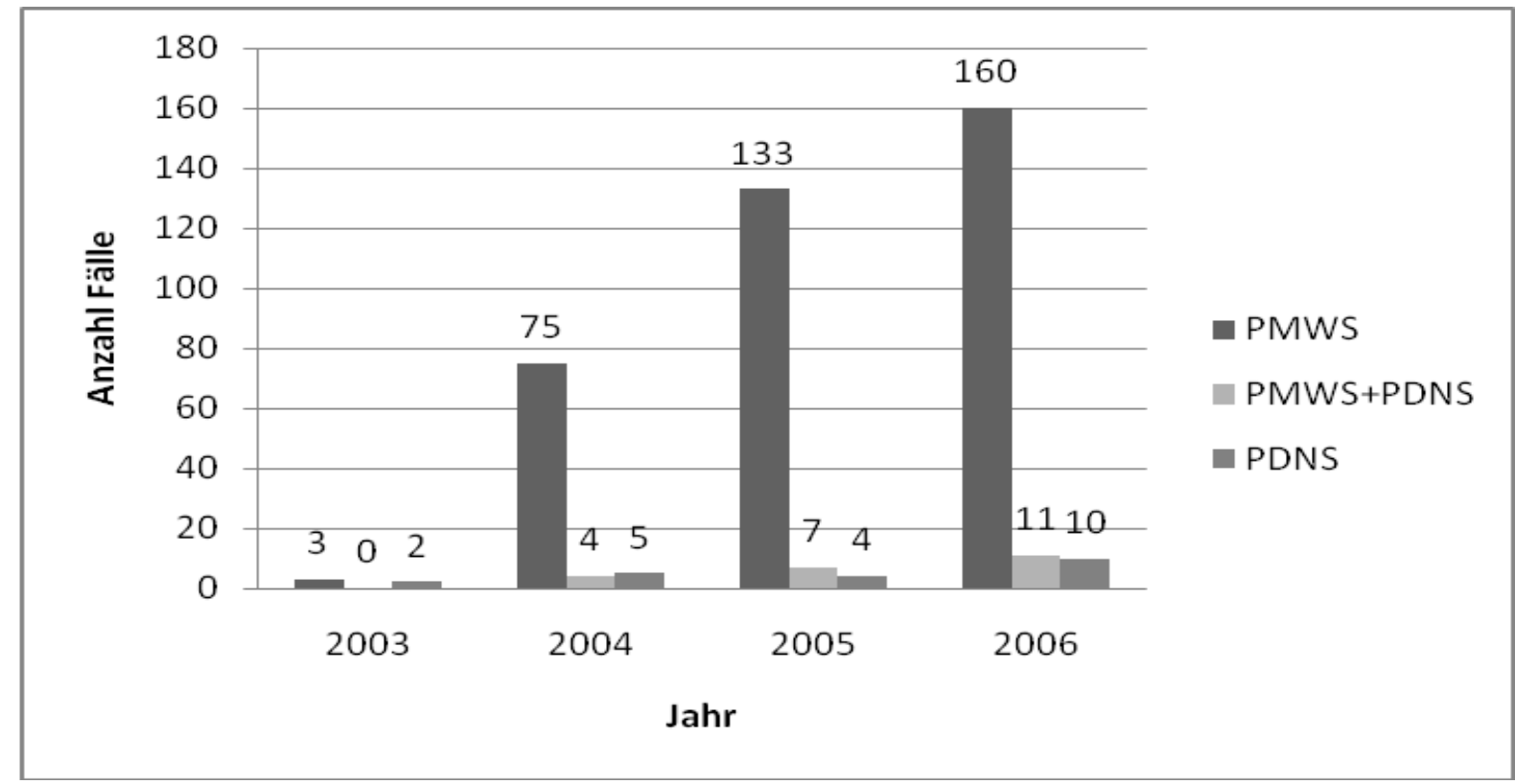

- PMWS

PMWS+PDNS

- PDNS 
604

605 Abbildung 2:

606

607

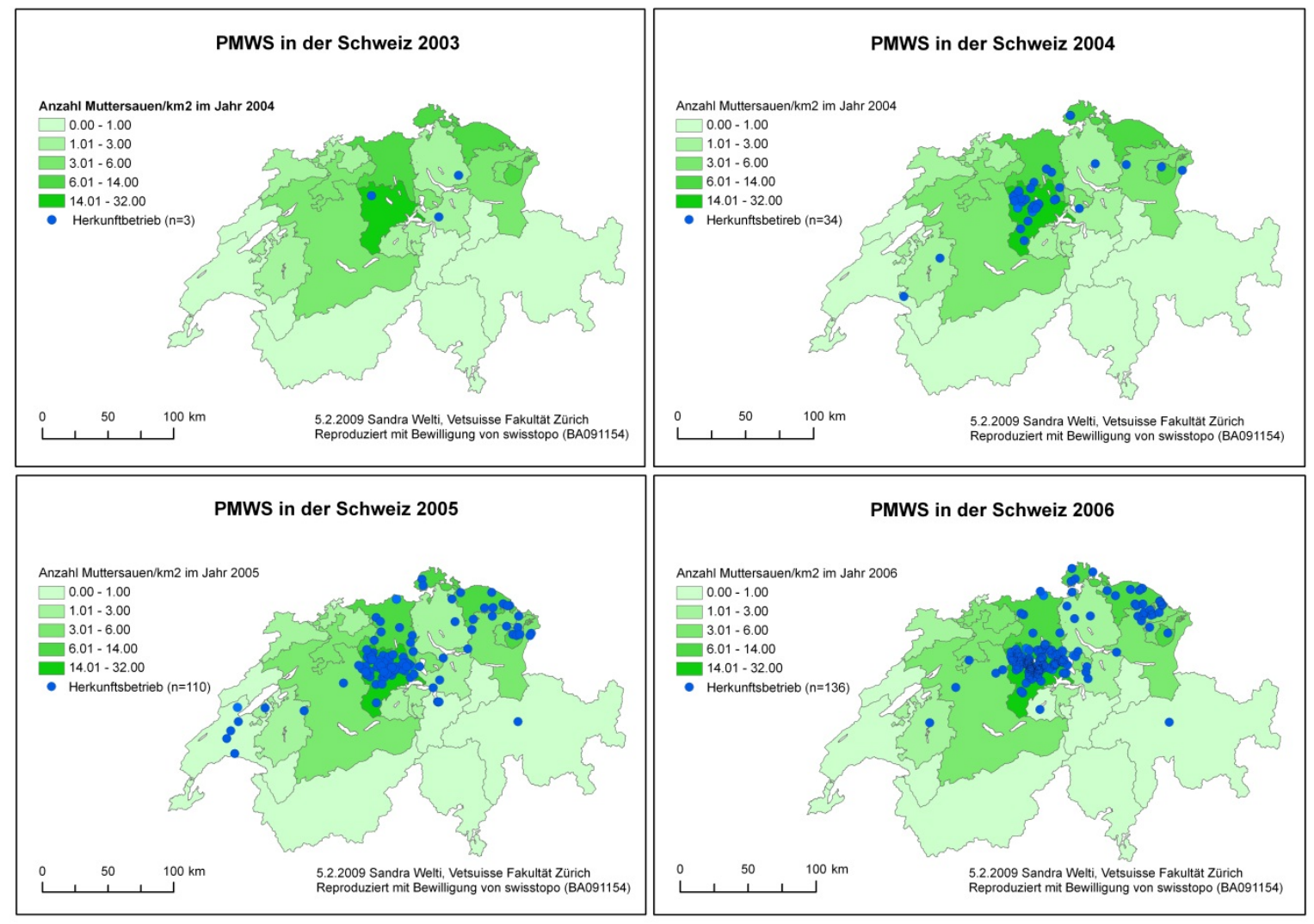

608

609

610

611

612

613

614

615

616

617

618

619

620

621

622 


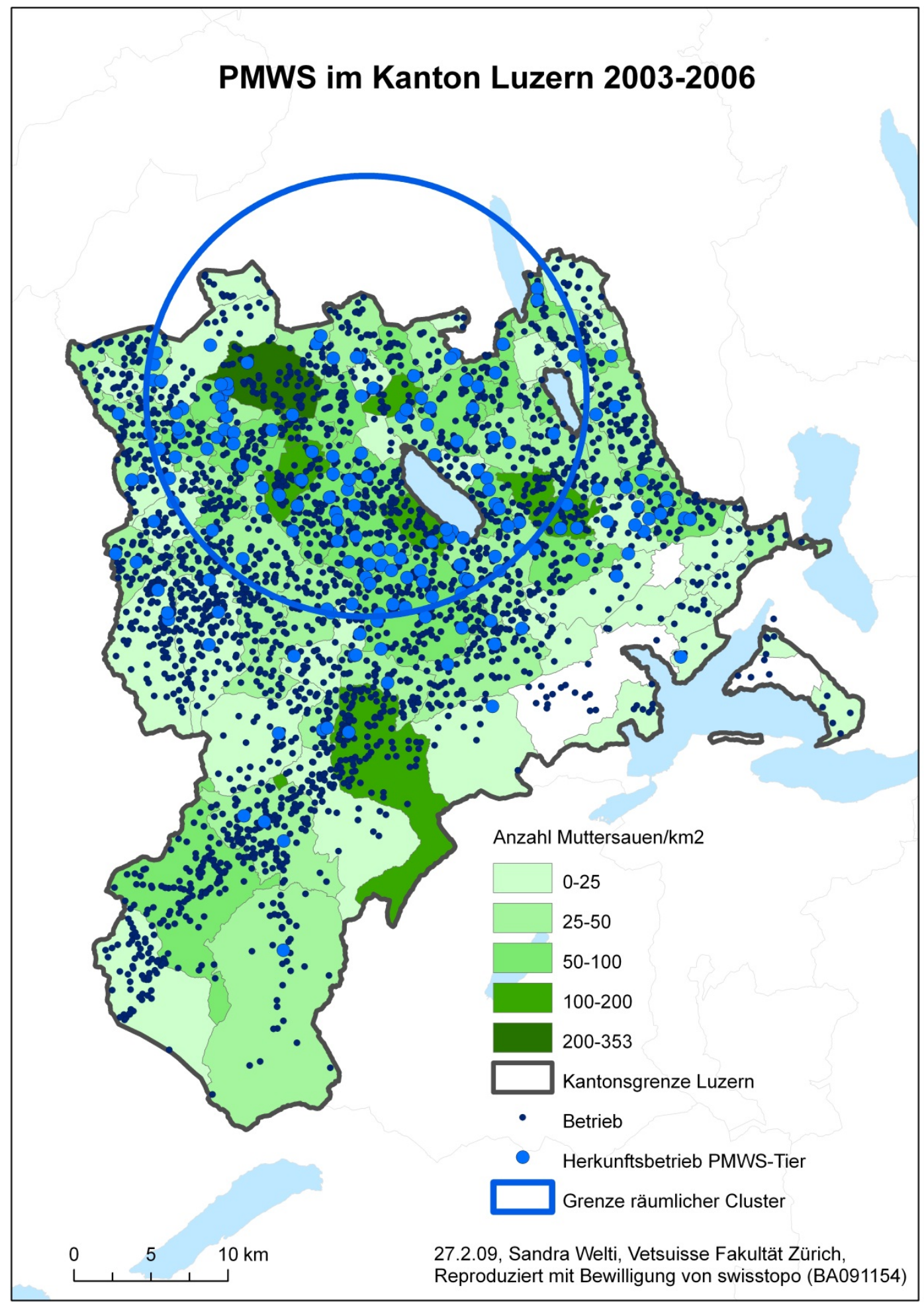


632 Abbildung 1: Anzahl PMWS-, PDNS- und Mischformen von PMWS\&PDNS633 Einzeltierdiagnosen.

634

635

636

637

638 Abbildung 2: Geographische Lage der Herkunftsbetriebe (॰) mit PMWS-Tieren in den 639 Jahren 2003 bis 2006.

640

641

642

643

644 Abbildung 3: Geographische Lage der Herkunftsbetriebe (॰) mit PMWS-Tieren und 645 Clusterberechnung in den Jahren 2003-2006 aus dem Kanton Luzern.

646 